\title{
Research on Consistency Judgement of Indication Error for Calibration Result of Humidity Sensor in Meteorology
}

\author{
Mingming Wei*, Fen Li, Jie Miao \\ Jiangxi Provincial Atmospheric Observation and Technical Center, Nanchang, China \\ Email: *weimingming@yeah.net
}

How to cite this paper: Wei, M.M., Li, F. and Miao, J. (2022) Research on Consistency Judgement of Indication Error for Calibration Result of Humidity Sensor in Meteorology. Atmospheric and Climate Sciences, 12, 43-53.

https://doi.org/10.4236/acs.2022.121004

Received: October 12, 2021

Accepted: November 27, 2021

Published: November 30, 2021

Copyright (c) 2022 by author(s) and Scientific Research Publishing Inc. This work is licensed under the Creative Commons Attribution International License (CC BY 4.0).

http://creativecommons.org/licenses/by/4.0/

\begin{abstract}
In order to solve the lack of relevant evaluation research on the accuracy of HMP155A humidity sensor calibration results in the past, this paper designs the corresponding experimental scheme, and obtains the corresponding calibration results according to the experimental scheme; Then the measurement uncertainty of the indication error in the calibration results is evaluated by GUM, and the corresponding extended uncertainty $U_{95}$ is obtained. Finally, according to the requirements of JJF1094-2016 characteristic evaluation of measuring instruments, combined with the calibration results and the actual situation of $U_{95}$, the conformity of the indication error of calibration is determined. The result is that each calibration point of the sensor meets the requirements of conformity determination and is within the qualified range. This research effectively makes up for the blank of the previous research on the conformity determination of the indication error of the calibration results and has strong theoretical and practical significance.
\end{abstract}

\section{Keywords}

Humidity Sensor, Calibration Results, Indication Error, Measurement

Uncertainty

\section{Preface}

Humidity represents the physical quantity of the degree of dryness of the atmosphere. At present, China's meteorological departments often use HMP155A humidity sensor for humidity detection. The accuracy of the detection data is of great significance for meteorological scientific research, climate change analysis and improving the ability of meteorological disaster prevention and reduction. In order to 
ensure the accuracy of the detection data of HMP155A humidity sensor, it is necessary to trace the quantity value regularly [1]. The quantity value tracing is generally in the way of calibration. The calibration method is reflected in the form of indication error and corresponding measurement uncertainty by comparing the humidity with the previous measurement standard. In order to ensure that the performance of the calibration results meets the standard, it is necessary to judge the conformity of the indication error of the measurement results according to the requirements of JJF1094-2016 characteristic evaluation of measuring instruments.

HMP155A humidity sensor is usually calibrated according to the humidity verification regulation. Since the verification regulation has made provisions on the evaluation method, measurement standard and environmental conditions, when the evaluated measuring instrument is in normal state, it is not necessary to judge the conformity of indication error according to the requirements of JJF1094-2016 evaluation of measuring instrument characteristics. However, further attention needs to be paid to whether the regulations in the meteorological department industry meet the relevant requirements. For example, the "verification regulation of meteorological platinum resistance temperature sensor in 2015" does not meet the requirements of instrument compliance assessment due to the accuracy grade of measuring instruments in the "verification regulation of meteorological platinum resistance temperature sensor in 2011". Therefore, in view of this situation, even if the verification regulation is used for calibration, it is still necessary to further determine and verify the compliance of indication error. In addition, the premise of instrument conformity determination is that the measuring instrument is in a normal state. However, during work, when the working state of the measuring instrument is unstable, the change is often very small, which is difficult for the staff to detect, which may also cause the results to fail to meet the requirements, At this time, the conformity judgment of the indication error of the calibration results can prompt whether the measuring instrument has faults that are difficult to detect. Lu T., et al. [2] and Zhang C., et al. [3] evaluated the uncertainty of humidity sensor. Yu Yanan, et al. [4], Sharma H., et al. [5], Martins L. L., et al. [6] and Hans I., et al. [7] analyzed the working principle of humidity sensor and calibration equipment, and Yang Z., et al. [8] and Wei, M., et al. [9] [10] evaluated the uncertainty of wind speed sensor, The above researchers either innovated the gum evaluation method or added the correlation analysis of different inputs in the $U_{95}$ uncertainty evaluation, which provides a good foundation for the follow-up research of this paper.

This paper will first calibrate HMP155A humidity sensor, then evaluate the measurement uncertainty of the indication error in the calibration results, and finally judge the conformity of the indication error of the calibration results according to the requirements of JJF1094-2016 evaluation of measuring instrument characteristics.

\section{Calibration Test}

The calibration method is generally based on the requirements of JJG (meteor- 
ology) 003-2011 verification regulation of humidity sensor in automatic weather station. The specific experimental method is as follows: firstly, put HMP155A humidity sensor and humidity standard (precision dew point instrument) into the humidity verification box, and set the temperature to $20^{\circ} \mathrm{C}$. Then, for $30 \% \%$ RH, 40\%\% RH, 55\%\% RH, 75\%\% RH and 95\% RH calibration points, first low humidity point by point to high humidity, and then from high humidity point by point to low humidity (one cycle calibration). After the humidity of each calibration point is stable, read the humidity sensor and humidity standard at the same time, and then determine its measurement performance by calculating the humidity error value of each calibration point. The calibration results are shown in Table 1.

When the calibration point is $95 \% \mathrm{RH}$, the MPEV (maximum allowable error value) of the humidity sensor is $\pm 8 \% \mathrm{RH}$, and the corresponding MPEV $= \pm 4 \%$ $\mathrm{RH}$ at other calibration points. The corresponding resolution of humidity sensor and standard is $0.01 \% \mathrm{RH}$, and the indication error of measurement results is generally reserved to $0.1 \% \mathrm{RH}$. By querying the superior traceability verification certificate of the humidity standard, it is known that the expanded uncertainty corresponding to each calibration point is $U=0.5 \% \mathrm{RH}(k=2)$. The experimental humidity sensor uses the $3 \mathrm{MS}$ system for reading. It is known from the test report of the humidity reading device of the 3 MS system (issued by the Chinese Academy of Metrology) that the digital multimeter is used to measure the DC voltage, And the measured voltage value is converted into the corresponding humidity output. The expanded uncertainty of digital multimeter is $U=0.0005$ $\mathrm{V}(k=2)$. See Table 2 for fluctuation, uniformity and correlation coefficient of temperature and humidity regulation box.

Table 1. Indication error of calibration results (Unit: \% RH).

\begin{tabular}{cccccccccccc}
\hline $\begin{array}{c}\text { Forward } / \\
\text { reverse stroke }\end{array}$ & \multicolumn{3}{c}{ Positive stroke } & \multicolumn{5}{c}{ Reverse stroke } \\
\hline Test point & 30 & 40 & 55 & 75 & 95 & 95 & 75 & 55 & 40 & 30 \\
1 & 1.27 & 1.20 & 0.92 & 0.40 & -0.16 & 0.60 & 0.43 & 0.86 & 1.24 & 1.19 \\
2 & 1.24 & 1.12 & 0.93 & 0.49 & -0.19 & 0.45 & 0.70 & 0.58 & 1.27 & 1.34 \\
3 & 1.24 & 1.17 & 0.97 & 0.41 & -0.24 & 0.16 & 0.64 & 0.81 & 1.12 & 1.12 \\
4 & 1.23 & 1.14 & 0.91 & 0.47 & -0.25 & 0.04 & 0.90 & 0.70 & 0.96 & 1.47 \\
5 & 1.24 & 1.11 & 0.94 & 0.49 & -0.25 & 0.15 & 0.63 & 0.80 & 1.19 & 1.45 \\
6 & 1.18 & 1.10 & 0.93 & 0.52 & -0.26 & 0.14 & 0.65 & 0.80 & 1.22 & 1.51 \\
7 & 1.11 & 1.16 & 0.91 & 0.54 & -0.22 & 0.05 & 0.65 & 0.72 & 1.24 & 1.29 \\
8 & 1.15 & 1.17 & 0.92 & 0.53 & -0.27 & -0.08 & 0.74 & 0.65 & 1.20 & 1.33 \\
9 & 1.14 & 1.16 & 0.96 & 0.54 & -0.23 & 0.24 & 0.74 & 0.88 & 1.21 & 1.51 \\
10 & 1.15 & 1.09 & 0.96 & 0.58 & -0.29 & 0.15 & 0.80 & 0.84 & 1.39 & 1.49 \\
Average value & 1.20 & 1.14 & 0.94 & 0.50 & -0.24 & 0.19 & 0.69 & 0.76 & 1.20 & 1.37 \\
\hline
\end{tabular}


Table 2. Fluctuation, uniformity and correlation coefficient of temperature and humidity regulating box.

\begin{tabular}{cccccc}
\hline $\begin{array}{c}\text { Calibration } \\
\text { point (\% RH) }\end{array}$ & 30 & 40 & 55 & 75 & 95 \\
Volatility (\% RH) & \pm 0.70 & \pm 74 & \pm 0.74 & \pm 0.45 & \pm 0.75 \\
Uniformity (\% RH) & {$[-0.41,0.41]$} & {$[-0.41,0.41]$} & {$[-0.42,0.42]$} & {$[-0.41,0.41]$} & {$[-0.47,0.47]$} \\
Correlation Coefficient & -0.09 & 0.13 & 0.51 & 0.26 & 0.20
\end{tabular}

\section{Evaluation of Measurement Uncertainty}

Measurement uncertainty, referred to as uncertainty, is an important index to measure the reliability of measurement results. Only when the measured value is given the corresponding uncertainty can it have practical significance. At present, the uncertainty evaluation is mainly based on the GUM method. The specific evaluation steps include: 1) establishing the corresponding measurement model and analyzing the source of the corresponding uncertainty; 2) Calculate the component value corresponding to the corresponding uncertainty source; 3 ) Calculate the combined standard uncertainty; 4) Calculate the extended uncertainty and determine the inclusion interval of the corresponding measurement results.

\subsection{Establishment of Measurement Model}

According to the calibration experiment, the corresponding theoretical measurement model is established as follows:

$$
\begin{aligned}
& \Delta H=\overline{H_{X}}-\overline{H_{S}} \\
& \overline{H_{X}}=\frac{1}{10} \sum_{i=1}^{10} H_{X i} \\
& \overline{H_{S}}=\frac{1}{10} \sum_{i=1}^{10} H_{S i}
\end{aligned}
$$

In order to ensure the accuracy of the experimental results, we usually read the standard humidity value and the measured humidity sensor value 10 times after each calibration point is stable, and then calculate the error of the corresponding average value. In Formula (1), $\overline{H_{X}}, \overline{H_{S}}$ and $\Delta H$ respectively represent the average value of 10 times of humidity sensor, 10 times of standard reading and the average value of corresponding 10 times of indication error. In formula (2), $H_{X i}$ and $H_{S i}$ respectively represent the indication values of single humidity sensor and humidity standard.

Considering that the measurement results are affected by some factors, combined with the actual measurement, the corresponding actual measurement model is established as follows:

$$
\Delta H=\overline{H_{X}}-\overline{H_{S}}+H_{1}+H_{2}+H_{3}+H_{4}+H_{5}+H_{6}+H_{7}
$$

$H_{1} \sim H_{7}$ are the introduction of standard resolution, standard uncertainty, hu- 
midity generator fluctuation, humidity generator uniformity, humidity sensor resolution, acquisition channel and rounding error respectively.

\subsection{Evaluation of Uncertainty Component}

GUM stipulates that the sources of general uncertainty can be divided into the uncertainty introduced by repetitive measurement (Type A standard uncertainty) and the uncertainty introduced by non-repetitive measurement (Type B standard uncertainty). Combined with the analysis of the experimental measurement model, $H_{X}$ and $H_{S}$ in Formula (3) are the uncertainty components introduced by repetitive measurement, corresponding to Type A standard uncertainty. $H_{1} \sim H_{7}$ are the uncertainty components introduced by non-repetitive measurement, corresponding to Type B standard uncertainty.

\subsubsection{Type A Standard Uncertainty}

According to the requirements of GUM, the type A standard uncertainty is the experimental standard deviation of the arithmetic mean of the corresponding independent repeatability measurement. When the number of repeated measurements is 10, Bessel formula is generally used to calculate the experimental standard deviation. Since the uncertainty components introduced by $H_{X}$ and $H_{S}$ for repeatability measurement can be expressed indirectly by $\Delta H$, the specific calculation formula of Type A standard uncertainty in this experiment is as follows [1]:

$$
u_{A}(x)=s(\bar{x})=\frac{s(x)}{\sqrt{10}}=\sqrt{\frac{\sum_{i=1}^{10} \Delta H_{i}}{10 \cdot(10-1)}}
$$

The relevant information of type A standard uncertainty of each calibration point obtained through calculation is shown in Table 3. The degree of freedom is the number of independent repeated measurements minus 1 , so the degree of freedom corresponding to type A uncertainty is 9 .

\subsubsection{Type B Standard Uncertainty}

According to the requirements of gum, the method of type B uncertainty is to judge the possible value interval $[x-a, x+a]$ of the measured value according to relevant information or experience. Assuming the probability distribution of the measured value and determining $k$ according to the probability distribution

Table 3. Evaluation results of type A standard uncertainty (Unit: \% RH).

\begin{tabular}{|c|c|c|c|c|c|c|}
\hline \multicolumn{2}{|c|}{ Calibration point } & 30 & 40 & 55 & 75 & 95 \\
\hline Positive & value & 0.0175 & 0.0113 & 0.0069 & 0.0183 & 0.0121 \\
\hline stroke & Degree of freedom & 9 & 9 & 9 & 9 & 9 \\
\hline Reverse & value & 0.0632 & 0.0392 & 0.0309 & 0.0347 & 0.0439 \\
\hline stroke & Degree of freedom & 9 & 9 & 9 & 9 & 9 \\
\hline
\end{tabular}


and the required probability $p$, the type B standard uncertainty can be obtained by the formula: $u_{\mathrm{B}}=a / k$. The corresponding relationship between the common distribution type probability $p$ and the inclusion factor $k$ is shown in Table 4 . When evaluating type B standard uncertainty, the degree of freedom [1] is:

$$
v=\frac{1}{2} \cdot\left[\frac{1}{1-\text { reliability }}\right]^{2}
$$

\section{1) Resolution introduction}

In Formula (3), $H_{1}$ and $H_{5}$ correspond to the resolution of humidity standard and humidity sensor respectively, and the values are $0.01 \% \mathrm{RH}$. The influence range of the measured value is $-0.005 \% \mathrm{RH}-0.005 \% \mathrm{RH}$, so $a=0.005 \% \mathrm{RH}$, and meets the uniformity distribution characteristics, so $k=1.732$. Since the value of the resolution does not change except for the failure of software and hardware, the reliability is estimated to be $100 \%$.

\section{2) Uncertainty of standard instrument value}

The uncertainty of the humidity standard corresponding to $\mathrm{H}_{2}$ in Formula (3) is obtained by querying the traceability certificate of the humidity standard. The corresponding measurement uncertainty at each measurement point is $U=0.5 \%$ $\mathrm{RH}, k=2$, which meets the normal distribution. The influence range of the measured value is $-0.5 \% \mathrm{RH}-0.5 \% \mathrm{RH}$, so $a=0.5 \% \mathrm{RH}$, and the reliability is estimated to be $80 \%$.

\section{3) Introduction of temperature and humidity control box}

In Formula (3), the fluctuation and uniformity of the temperature regulation and commissioning box corresponding to $H_{3}$ and $H_{4}$ are shown in Table 2 . Taking the $55 \% \mathrm{RH}$ calibration point as an example, its volatility is $\pm 0.74 \% \mathrm{RH}$, and the influence range of the measured value is corresponding to $-0.74 \% \mathrm{RH}$ $0.74 \% \mathrm{RH}$, so $a=0.74 \% \mathrm{RH}$, and meets the arcsine distribution characteristics, with the corresponding $k=1.414$; The uniformity is $[-0.42,0.42]$, and the influence range of the measured value is corresponding to $-0.42 \% \mathrm{RH}-0.42 \% \mathrm{RH}$, so $a=0.5 \% \mathrm{RH}$, and meets the uniformity distribution characteristics, so $k=$ 1.732. The reliability estimates corresponding to volatility and uniformity are both $80 \%$.

\section{4) Introduction of collector channel error}

According to the error introduced by the internal acquisition channel of the 3 MS device corresponding to $H_{6}$ in Formula (3), the humidity reading device of the $3 \mathrm{MS}$ system uses a multimeter to read the voltage value of the temperature sensor, and then converts it into the humidity value. By querying the traceability certificate of the corresponding multimeter, the corresponding uncertainty in

Table 4. Correspondence between $p$ and $k$ of common probability distributions.

\begin{tabular}{ccccc}
\hline Distribution type & Uniform & Arcsine & Normal & Normal \\
\hline Probability $P$ & $100 \%$ & $100 \%$ & $95 \%$ & $95.45 \%$ \\
Inclusion factor $k$ & 1.732 & 1.414 & 1.96 & 2 \\
\hline
\end{tabular}


the range range is: $U=0.0005 \mathrm{~V}, k=2$. Because the voltage of $(0-1) \mathrm{V}$ corresponds to the humidity of $(0-100) \% \mathrm{RH}$, The corresponding conversion relationship is: $0.0005 \mathrm{~V} / 100 \mathrm{~V}(\% \mathrm{RH})^{-1}=0.05 \% \mathrm{RH}$, the influence range of the measured value is $-0.05 \% \mathrm{RH} \sim 0.05 \% \mathrm{RH}$, so $a=0.05 \% \mathrm{RH}$, which meets the normal distribution, and the reliability is estimated to be $90 \%$.

\section{5) Rounding error introduction}

The rounding error corresponding to $H_{7}$ in Formula (3) is introduced. According to previous experience, the rounding error is $0.1 \% \mathrm{RH}$, the error range is- $0.05 \% \mathrm{RH}-0.05 \% \mathrm{RH}, a=0.05 \% \mathrm{RH}$, which meets the uniformity distribution, and the reliability is estimated to be $90 \%$.

\subsubsection{Evaluation Results of Standard Uncertainty Component}

Based on the above analysis of uncertainty components, the corresponding uncertainty component values are calculated according to GUM's rules on uncertainty component calculation. Taking $55 \% \mathrm{RH}$ as an example, the values and relevant information are shown in Table 5.

\subsection{Uncertainty Evaluation of Combined Standard}

According to the requirements of GUM, the synthetic standard uncertainty is expressed in $u_{c}$. When the measurement model is linear equation, its calculation formula [1] is:

$$
u_{\mathrm{C}}=\sqrt{\sum_{i=1}^{N} u^{2}\left(x_{i}\right)+2 \sum_{i=1}^{N-1} \sum_{j=1+1}^{N} r\left(x_{i}, x_{j}\right) u\left(x_{i}\right) u\left(x_{j}\right)}
$$

In combination with the actual situation of formula (3) of the measurement model, $\overline{H_{X}}-\overline{H_{S}}$ corresponds to Type A standard uncertainty $u_{\mathrm{A}}, H_{1}-H_{7}$ corresponds to type B standard uncertainty $u_{1}-u_{7}$ respectively, and because the corresponding uniformity of the temperature and humidity control box will be affected when it fluctuates, there is a certain correlation between the fluctuation and uniformity of the temperature and humidity control box, and the corresponding correlation coefficient is $r_{34}=0.51$ (Table 2), Then the synthetic standard

Table 5. Relevant information of uncertainty component at 55\% RH calibration point.

\begin{tabular}{cccccccc}
\hline \multirow{2}{*}{ component } & Source of uncertainty & $\begin{array}{c}\text { probability } \\
\text { distribution }\end{array}$ & $\begin{array}{c}\boldsymbol{a}(\% \\
\mathrm{RH})\end{array}$ & $\boldsymbol{k}$ & $\begin{array}{c}\text { Free } \\
\text { degree }\end{array}$ & $\begin{array}{c}\text { reliability } \\
\text { Component value } \\
(\% \mathrm{RH})\end{array}$ \\
\hline$u_{\mathrm{A}}$ & Type A standard uncertainty & Normal & -- & -- & 9 & -- & 0.0069 \\
$u_{1}$ & Resolution of standard sensor & Uniformity & 0.005 & 1.732 & $\infty$ & $100 \%$ & 0.0029 \\
$u_{2}$ & Accuracy of standard sensor & Normal & 0.500 & 2 & 12.5 & $80 \%$ & 0.2500 \\
$u_{3}$ & temperature and Fluctuation & Arcsine & 0.745 & 1.414 & 12.5 & $80 \%$ & 0.5268 \\
$u_{4}$ & humidity control box Uniformity & Uniformity & 0.418 & 1.732 & 12.5 & $80 \%$ & 0.2416 \\
$u_{5}$ & Resolution of measuring sensor & Uniformity & 0.005 & 1.732 & 50 & $90 \%$ & 0.0029 \\
$u_{6}$ & Error of collector channel & Normal & 0.050 & 2 & 50 & $90 \%$ & 0.0250 \\
$u_{7}$ & Rounding error & Uniformity & 0.050 & 1.732 & 50 & $90 \%$ & 0.0289
\end{tabular}


uncertainty $u_{\mathrm{c}}$ is expressed as:

$$
u_{\mathrm{C}}=\sqrt{u_{\mathrm{A}}^{2}+\sum_{i=3}^{7} u_{i}^{2}+r_{34} \cdot u_{3} \cdot u_{4}}
$$

In addition, due to the mutual inclusiveness between the uncertainty component introduced by repeatability measurement and the uncertainty component introduced by resolution, it is necessary to compare the corresponding magnitude and discard the components with a small magnitude.

Since the magnitude of type a standard uncertainty is greater than the standard uncertainty measurement introduced by resolution, the values of $u_{1}$ and $u_{5}$ are discarded in the calculation of $u_{c}$. Formula (7) is transformed into the corresponding calculation formula:

$$
u_{\mathrm{C}}=\sqrt{u_{\mathrm{A}}^{2}+u_{2}^{2}+u_{3}^{2}+u_{4}^{2}+r_{34} \cdot u_{3} \cdot u_{4}+u_{6}^{2}+u_{7}^{2}}
$$

In $u_{c}$ calculation, the calculation formula of the corresponding effective degrees of freedom [1] is:

$$
v_{\text {eff }}=\frac{u_{\mathrm{c}}^{4}}{\sum_{i=i}^{N} \frac{u_{i}^{4}}{v_{i}^{4}}}
$$

\subsection{Expanded Uncertainty and Measurement Results}

The expanded uncertainty is generally expressed by $U$, and its value calculation formula is $U=k \times u_{c}, k=2$ is generally selected without special instructions, and the corresponding inclusion probability is $p=95.45 \%$. Since the expanded uncertainty (represented by $U_{95}$ ) when $p=95 \%$ is required for compliance determination in this study, the corresponding inclusion factor $k_{95}$ needs to be calculated separately, which needs to be obtained by querying the t-value distribution table according to the value of the effective degree of freedom $v_{\text {eff }}\left(k_{95}=1.96\right.$, only when $v_{\text {eff }}=\infty$ ). The uncertainty evaluation results and relevant information obtained through calculation are shown in Table 6.

\section{Determination of Conformity of Indication Error}

According to the requirements of JJF1094-2016 characteristic evaluation of measuring instruments, when judging the conformity of indication error in calibration results, there are the following four situations:

1) When $U_{95} \leq \mathrm{MPEV} / 3$ (MPEV is the maximum allowable error), it meets the compliance judgment requirements of indication error $|\delta|<\mathrm{MPEV}$ is qualified ( $\delta$ Is the indication error), otherwise it is judged as unqualified.

2) When $U_{95}>\mathrm{MPEV} / 3$, whether it meets the compliance determination requirements of indication error is divided into two cases. The first is when: $|\delta|>$ $\mathrm{MPEV}+U_{95}$ is not up to the conformity determination requirements of indication error, and it is directly determined as unqualified at this time; Second: when $|\delta| \leq \mathrm{MPEV}-U_{95}$, meeting the conformity judgment requirements of indication error, it is judged as qualified at this time; 
Table 6. Information related to uncertainty evaluation results.

\begin{tabular}{ccccccc}
\hline Calibration point & $\begin{array}{c}\text { Indication } \\
\text { error } \delta\end{array}$ & $\boldsymbol{u}_{\mathrm{c}}$ & $\begin{array}{c}\text { Effective degree } \\
\text { of freedom }\end{array}$ & $\boldsymbol{k}_{95}$ & $\boldsymbol{U}_{95}$ \\
\hline & 30 & 1.2 & 0.59 & 24 & 2.0639 & 1.2 \\
Increase & 40 & 1.1 & 0.65 & 23 & 2.0687 & 1.4 \\
humidity & 55 & 0.9 & 0.73 & 23 & 2.0687 & 1.5 \\
& 75 & 0.5 & 0.38 & 29 & 2.0452 & 0.8 \\
& 95 & -0.2 & 0.69 & 24 & 2.0639 & 1.4 \\
\hline \multirow{2}{*}{ Reduce } & 95 & 0.2 & 0.70 & 24 & 2.0639 & 1.4 \\
humidity & 75 & 0.7 & 0.38 & 29 & 2.0452 & 0.8 \\
& 55 & 0.8 & 0.73 & 23 & 2.0687 & 1.5 \\
& 40 & 1.2 & 0.65 & 23 & 2.0687 & 1.4 \\
& 30 & 1.4 & 0.59 & 24 & 2.0639 & 1.2 \\
\hline
\end{tabular}

$\delta$ For the indication error of the corresponding humidity calibration result, the effective degree of freedom is obtained by formula (9), $k_{95}$ is obtained by querying the T value distribution table of the effective degree of freedom, $u_{c}$ is obtained by formula (8), and the final $U_{95}$ is obtained by the product of $k_{95}$ and $u_{c}$. The final $U_{95}$ value is to prepare for the subsequent determination of the conformity of indication error.

Table 7. Determination of conformity of indication error.

\begin{tabular}{ccccccc}
\hline \multicolumn{2}{c}{ Calibration point } & $\mathbf{3 0}$ & $\mathbf{4 0}$ & $\mathbf{5 5}$ & $\mathbf{7 5}$ & $\mathbf{9 5}$ \\
\hline \multirow{2}{*}{$\begin{array}{c}\text { Positive } \\
\text { stroke }\end{array}$} & $U_{95} \leq 1 / 3 \mathrm{MPEV}$ & $\mathrm{YES}$ & $\mathrm{NO}$ & $\mathrm{NO}$ & YES & YES \\
& $\delta$ & 2.8 & 2.6 & 2.5 & 3.2 & 6.6 \\
\multirow{2}{*}{$\begin{array}{c}\text { Reverse } \\
\text { stroke }\end{array}$} & $\mathrm{MPEV}-U_{95}$ & 1.2 & 1.1 & 0.9 & 0.5 & -0.2 \\
\hline
\end{tabular}

Note: when $95 \% \mathrm{RH}, \mathrm{MPEV}=8 \% \mathrm{RH}$; At other calibration points, $\mathrm{MPEV}=4 \% \mathrm{RH}$.

3) When $U_{95}>\mathrm{MPEV} / 3, \mathrm{MPEV}-U_{95}<\delta<\mathrm{MPEV}+U_{95}$, to be determined. In this case, it is generally recommended to recheck the equipment, observe whether there are abnormalities, and then calibrate again. Table 7 shows the relevant information of value error determination.

According to the above criteria, we can conclude that no matter how large the value of u95 is, as long as $|\delta| \leq \mathrm{MPEV}-U_{95}$, then they all meet the compliance determination requirements and are determined as qualified. It can be seen from Table 7 that all calibration points meet the requirements $|\delta| \leq \mathrm{MPEV}-U_{95}$, so the judgment results are qualified.

\section{Conclusion}

In this paper, a HMP155A humidity sensor is selected for calibration experiment 
with reference to the verification regulation. The measurement uncertainty is analyzed and evaluated according to the indication error in the calibration results, and the corresponding extended uncertainty $U_{95}$ is obtained. Finally, the conformity determination of indication error is completed according to the requirements of JJF1094-2016 evaluation of measuring instrument characteristics and the calculation results of $U_{95}$. Through experiments, it is concluded that the HMP155A humidity sensor meets the conformity judgment requirements of indication error at each calibration point, and the conclusions are qualified. This study effectively makes up for the blank of no compliance judgment on the indication error of the calibration results in the calibration of humidity sensor in the past and gives the corresponding technical route, which has strong theoretical and practical significance.

\section{Acknowledgments}

This work is supported by the National Natural Science Foundation of China under Grant No. 42065011.

\section{Conflicts of Interest}

The authors declare no conflicts of interest regarding the publication of this paper.

\section{References}

[1] (2008) ISO/IEC Guide 98-3: 2008, Uncertainty of Measurement-Part 3: Guide to the Expression of Uncertainty in Measurement (GUM).

[2] Lu, T. and Chen, C. (2007) Uncertainty Evaluation of Humidity Sensors Calibrated by Saturated Salt Solutions. Measurement, 40, 591-599. https://doi.org/10.1016/j.measurement.2006.09.012

[3] Zhang, C., Zhang, W., Webb, D.J., et al. (2010) Optical Fibre Temperature and Humidity Sensor. Electronics Letters, 46, 643-644. https://doi.org/10.1049/el.2010.0879

[4] Yu, Y., et al. (2021) Multi-Channel Impedance Measurement System for Polymer Humidity Sensors. Measurement, 176, Article No. 109113. https://doi.org/10.1016/j.measurement.2021.109113

[5] Sharma, H., Vaidya, U. and Ganapathysubramanian, B. (2019) A Transfer Operator Methodology for Optimal Sensor Placement Accounting for Uncertainty. Building and Environment, 155, 334-349. https://doi.org/10.1016/j.buildenv.2019.03.054

[6] Martins, L.L., Ribeiro, A.S., e Sousa. J.A., et al. (2012) Measurement Uncertainty of Dew-Point Temperature in a Two-Pressure Humidity Generator. International Journal of Thermophysics, 33, 1568-1582. https://doi.org/10.1007/s10765-011-1005-Z

[7] Hans, I., Burgdorf, M., Buehler, S.A., et al. (2019) An Uncertainty Quantified Fundamental Climate Data Record for Microwave Humidity Sounders. Remote Sensing, 11, Article No. 548. https://doi.org/10.3390/rs11050548

[8] Yang, Z., Zhang, H., Wang, Q., et al. (2021) Uncertainty Evaluation of Wind Speed Sensor in Automatic Weather Station. IOP Conference Series: Earth and Environmental Science, 768, Article No. 012008. https://doi.org/10.1088/1755-1315/768/1/012008 
[9] Wei, M., Zeng, Y., Wen, C., et al. (2019) Comparison of MCM and GUM Method for Evaluating Measurement Uncertainty of Wind Speed by Pitot Tube. MAPAN, 34, 345-355. https://doi.org/10.1007/s12647-019-00339-3

[10] Wei, M., Zeng, Y., Zou, L., et al. (2019) Analysis of the Influence of Water-Vapor Correction Term on the Measurement Uncertainty of Wind Speed. MAPAN, 34, 333-343. https://doi.org/10.1007/s12647-019-00338-4 